SLAC-PUB-8397

March, 2000

\title{
POLARIMETRY AT A FUTURE LINEAR COLLIDER - HOW PRECISE?
}

\author{
M. WOODS* \\ Stanford Linear Accelerator Center \\ Stanford University, Stanford, CA 94309, USA
}

\begin{abstract}
At a future linear collider, a polarized electron beam will play an important role in interpreting new physics signals. Backgrounds to a new physics reaction can be reduced by choice of the electron polarization state. The origin of a new physics reaction can be clarified by measuring its polarization-dependence. This paper examines some options for polarimetry with an emphasis on physics issues that motivate how precise the polarization determination needs to be. In addition to Compton polarimetry, the possibility of using Standard Model asymmetries, such as the asymmetry in forward W-pairs, is considered as a possible polarimeter. Both $e^{+} e^{-}$and $e^{-} e^{-}$collider modes are considered.
\end{abstract}

Presented at

$$
e^{-} e^{-99}
$$

3rd International Workshop on Electron-Electron Interactions at Te $V$ Energies

December 10-12, 1999

UC Santa Cruz, Santa Cruz, CA, USA

*Work supported by the Department of Energy, Contract DE-AC03-76SF00515. 


\section{Introduction}

This paper addresses the issue of how accurately one should measure the electron beam polarization, $P_{e}$, at a future linear collider. The collider performance parameters shown in Table 1 are used. I begin by considering how accurately Standard Model (SM) asymmetries may be measured, and the requirements these measurements place on the polarimetry. In addition to Compton polarimetry 1 the measurement of SM physics asymmetries for polarimetry is considered. This type of polarimetry has previously been proposed when both colliding beams are polarized 2.3 Here, I additionally consider the possibility to use the asymmetry in forward $\mathrm{W}$-pairs in $e^{+} e^{-}$collisions, when only the electron beam is polarized.

The precision of the polarimetry can affect the discovery potential for a new physics signal. I examine the importance of precise polarimetry for accurately assessing W-pair backgrounds.

Beam-beam effects in the collision process have a significant impact on polarimetry. First, significant depolarization can result, and one needs to determine how the measured $P_{e}$ is related to the luminosity-weighted polarization, $P_{e}^{\text {lum }}$. At high luminosities with large beamsstrahlung disruption, it may no longer be possible to place a Compton polarimeter in the extraction line from the Interaction Region (IR). This will limit the ability to accurately determine the amount of depolarization in the collision process.

Table 1. Collider performance parameters for this study.

\begin{tabular}{ccc}
\hline Parameter & $e^{+} e^{-}$ & $e^{-} e^{-}$ \\
\hline$\sqrt{(s)}$ & $500 \mathrm{GeV}$ & $500 \mathrm{GeV}$ \\
$\int L d t$ & $80 \mathrm{fb}^{-1}$ & $25 \mathrm{fb}^{-1}$ \\
$P_{1}$ & $90 \%$ & $90 \%$ \\
$P_{2}$ & $0 \%$ & $90 \%$ \\
\hline
\end{tabular}

\section{Standard Model Asymmetries in $e^{+} e^{-}$}

For an $e^{+} e^{-}$collider with $500 \mathrm{GeV}$ center-of-mass energy, the dependence of SM production cross sections on $P_{e}$ is plotted in Figure 1. This Figure is taken from a Report for Snowmass 1996 on the Next Linear Colliden and assumes the positron beam is unpolarized. Following the study in that Report, some SM asymmetries are estimated for a detector with an acceptance of $|\cos \theta|<0.99$, for an integrated luminosity of $80 \mathrm{fb}^{-1}$. These are summarized in Table 2. The left-right asymmetry, $A_{L R}$, and its statistical and systematic uncertainties are given by 


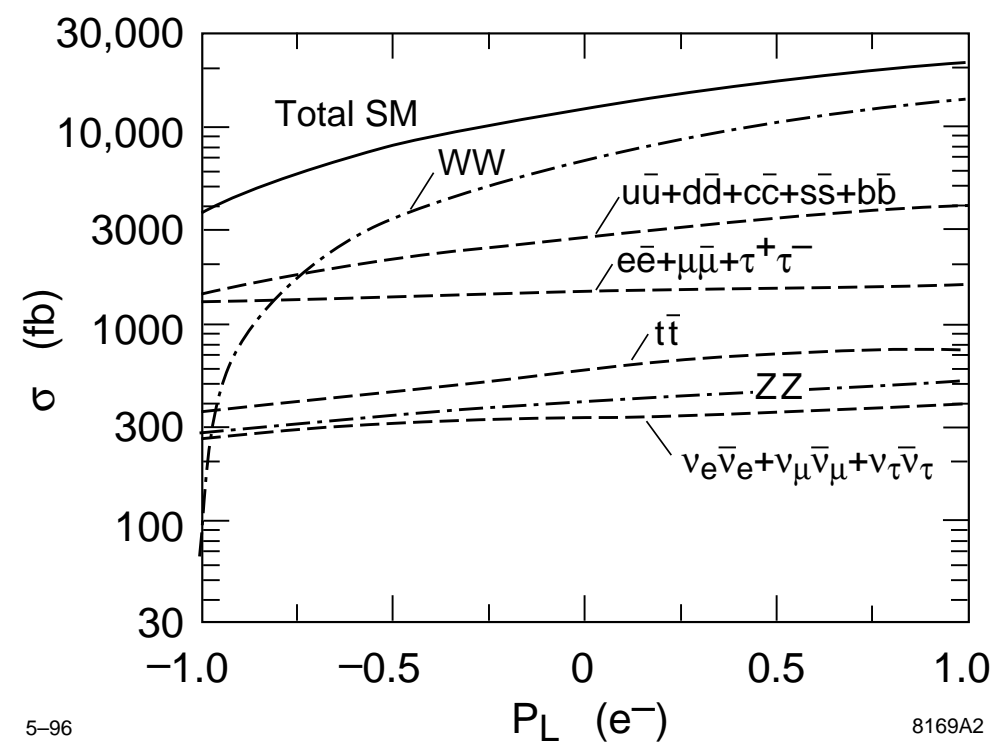

Fig. 1. Dependencg of Standard Model cross sections on the electron beam polarization at a 500 $\mathrm{GeV} e^{+} e^{-}$collider.

$$
\begin{aligned}
A_{L R} & =\frac{\left(\sigma_{L}-\sigma_{R}\right)}{\left(\sigma_{L}+\sigma_{R}\right)} \\
\delta A_{L R} & =\sqrt{\frac{\left[1-\left(P A_{L R}\right)^{2}\right]}{P^{2} N}+\left(\frac{\delta P}{P}\right)^{2} A_{L R}^{2}} \\
& =\sqrt{\left(\delta A_{L R}^{\text {stat }}\right)^{2}+\left(\delta A_{L R}^{\text {syst }}\right)^{2}},
\end{aligned}
$$

where $P=90 \%$ is the electron polarization; $N_{L}\left(N_{R}\right)$ is the number of observed events with the left-(right-)polarized beam, and $N=N_{L}+N_{R}$. Equal integrated luminosities are accumulated with the left- and right-polarized electron beam.

Table 2. Standard Model Production Asymmetries.

\begin{tabular}{cccc}
\hline Final State & \# events & $A_{L R}$ & $\frac{\delta A_{L R}^{\text {stat }}}{A_{L R}}$ \\
\hline$W^{+} W^{-}$ & $560 \mathrm{~K}$ & $99.2 \%$ & 0.0007 \\
$q \bar{q}$ & $250 \mathrm{~K}$ & $45 \%$ & 0.005 \\
$l^{+} l^{-}$ & $120 \mathrm{~K}$ & $10 \%$ & 0.032 \\
\hline
\end{tabular}

Table 2 indicates that it is necessary to have better than $1 \%$ polarimetry to fully exploit testing SM predictions for these asymmetry measurements. This can be achieved with a precise Compton polarimeter. It is also interesting to consider whether the asymmetry in W-pairs could be used as a polarimeter. The Feynman diagrams contributing to $\mathrm{W}$-pair production are shown in Figure 2. The diagram in Figure 2 $\mathrm{b}$ is highly suppressed. In the forward detector regions $(\cos \theta>0.7)$, the 
a)

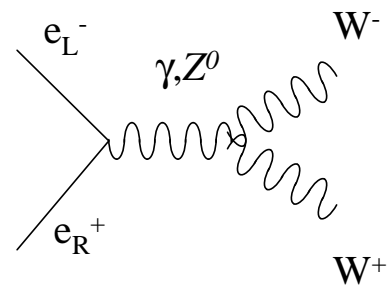

b)

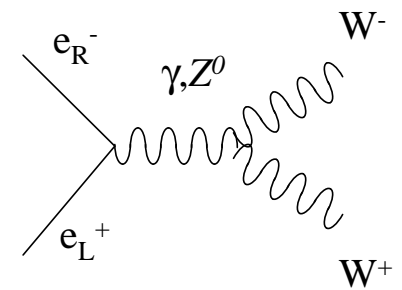

c)

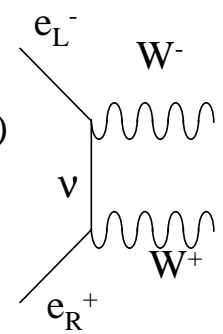

Fig. 2. Feynman diagrams for $\mathrm{W}$-pair production at an $e^{+} e^{-}$collider.

exchange diagram in Figure 22c dominates and $A_{L R}$ for W-pairs is essentially $100 \%$. The measured left-right asymmetry in forward W-pairs can therefore be used to determine the electron beam polarization. ${ }^{a}$ To achieve sub-1\% accuracy for $P_{e}$ will require achieving backgrounds to the $\mathrm{W}$-pair sample below $1 \%$. This has been achieved at LEP200 for the $\mathrm{W}$ mass measurements, where one of the $\mathrm{Ws}_{\mathrm{is}}$ is required to decay to $\mu \nu_{\mu}$ and tight cuts are placed on the reconstructed $\mathrm{W}$ mass 6 A detailed study needs to be done to determine whether this low background could also be achieved with the forward detector regions at a linear collider. An advantage of using a detector physics asymmetry for polarimetry rather than Compton polarimetry is that $P_{e}^{\text {lum }}$ is directly determined and beam-beam depolarization effects are properly accounted for. ${ }^{b}$

A wonderful example of the physics possibilities with precise asymmetry measurements is the linear collider $Z^{0}$-Factory option. It is very desirable to accumulate a large $Z^{0}$ sample ( $>10$ million) with a polarized electron beam. For example, Ref. 7 considers achieving a sample of $10^{9} Z^{0}$ decays with $80 \%$ electron polarization and $60 \%$ positron polarization. This enables the determination of the weak mixing angle, by measuring the polarization-dependent cross sections for $Z^{0}$ production, with an unprecedented accuracy of $\delta \sin ^{2}\left(\theta_{W}^{e f f}\right)=1.3 \cdot 10^{-5}$. In this case,

\footnotetext{
${ }^{a}$ One will still be able to check for new physics processes that might affect this polarization determination, by measuring the dependence of the result on the polar scattering angle.

${ }^{b}$ If depolarization effects are significant, however, there may be a significant dependence of $P_{e}$ on the electron's effective collision energy. In this case, the energy distribution of collision electrons in the W-pair sample should be the same as the distribution of collision electrons in the physics sample being studied to ensure that $P_{e}^{l u m}$ is being accurately determined.
} 
the availability of a polarized positron beam allows for very precise polarimetry 2 For the case where the positron beam is not polarized, it will still be very desirable to make a significant improvement on the SLD weak mixing angle measurement, $\delta \sin ^{2}\left(\theta_{W}^{e f f}\right)=2.8 \cdot 10^{-4}$ This will require better than $0.5 \%$ polarimetry from a Compton polarimeter located in the extraction line from the IR. One can also hope to resolve whether the anomalies observed in the $Z b \bar{b}$ asymmetry measurement $\$$ at SLD and LEP are due to statistical fluctuations, systematic problems or new physics.

\section{Standard Model Asymmetries in $e^{-} e^{-}$}

For the linear collider operating with $e^{-} e^{-}$collisions, both beams can be polarized. One of the important measurements that will be made is an accurate measurement of the weak mixing angle away from the $Z^{0}$-pole, by measuring the polarization-dependent cross sections in Moller scattering $\left(e^{-} e^{-} \rightarrow e^{-} e^{-}\right)$. With both beams polarized, one can measure three independent asymmetries which can be chosen to be

$$
\begin{aligned}
& A_{1}=\frac{N_{L L}-N_{R R}}{N_{L L}+N_{R R}} \\
& A_{2}=\frac{N_{R R}-N_{L R}}{N_{R R}+N_{L R}} \\
& A_{3}=\frac{N_{L R}-N_{R L}}{N_{L R}+N_{R L}} .
\end{aligned}
$$

From these asymmetry measurements, one can determine $\sin ^{2}\left(\theta_{W}^{e f f}\right), P_{1}$, and $P_{2}$. A detailed study of this has been done in Ref. 3 for a $500 \mathrm{GeV}$ collider, beam polarizations of $90 \%$, detector acceptance with $|\cos \theta|<0.995$, and integrated luminosity of $25 \mathrm{fb}^{-1}$. They find that the beam polarizations can be determined with an accuracy of $0.9 \%$, and that the weak mixing angle can be determined with an accuracy of $\delta \sin ^{2}\left(\theta_{W}^{e f f}\right)=0.00026$. This accuracy is comparable to that achieved with SLD's $A_{L R}$ measurement at the $Z^{0}$-pole and will be the best measurement of the weak mixing angle away from the $Z^{0}$-pole. The running of $\sin ^{2}\left(\theta_{W}^{e f f}\right) 9$ with $Q^{2}$ will be measured with excellent precision, a factor 3 better than that expected from the SLAC E158 experiment. 10 Excellent sensitivity to additional $Z^{0}$ bosons (up to $m_{Z^{\prime}} \approx 10 \mathrm{TeV}$ ) and to electron compositeness (up to a compositeness scale, $\Lambda \approx 100 \mathrm{TeV}$ ) will be achieved. The beam polarization uncertainty is comparable to what one can expect with a Compton polarimeter, and has the advantage that it directly measures $P_{e}^{\text {lum }}$. The determination of $P_{e}^{\text {lum }}$ from the Moller scattering analysis can also be applied to other physics analyses. ${ }^{c}$

\footnotetext{
${ }^{c}$ One may have to make small corrections for the dependence of $P_{e}^{\text {lum }}$ on the energy distribution of the collision electrons if depolarization effects are significant.
} 


\section{Background Suppression of $\mathrm{W}$-pairs}

For the $e^{+} e^{-}$collider, W-pair background will be an obstacle for observing new physics reactions. Beam polarization will be an important tool for understanding and reducing this background. The production cross section for $\mathrm{W}$-pairs may be written as

$$
\sigma\left(P_{1}, P_{2}\right)=\frac{1}{4}\left[\left(1-P_{1}\right)\left(1+P_{2}\right) \sigma_{L R}+\left(1+P_{1}\right)\left(1-P_{2}\right) \sigma_{R L}\right],
$$

where $P_{1}$ is the electron beam polarization; $P_{2}$ is the positron beam polarization; and $\sigma_{L R}\left(\sigma_{R L}\right)$ is the W-pair production cross section for a left-(right-)handed electron colliding with a right-(left-)handed positron. As noted in Ref. 11, $\sigma_{R L}$ is highly suppressed, $\left(\sigma_{R L} / \sigma_{L R} \approx 0.004\right)$.

For assessing the utility of achieving high beam polarizations, it is useful to construct a Figure-of-Merit (FOM) defined as the ratio, $R=\sigma\left(P_{1}, P_{2}\right)^{\max } / \sigma\left(P_{1}, P_{2}\right)^{\text {min }}$ where the maximum (minimum) W-pair production cross section is achieved with a left-(right-)polarized electron beam and a right-(left-)polarized positron beam. Table 3 summarizes this FOM for some possibilities for the beam polarizations. It is desirable to achieve a high electron beam polarization, since the FOM increases by a factor of 2 when improving $P_{1}$ from $80 \%$ to $90 \%$. The utility of polarizing

the positron beam is also evident,11 though this may be difficult to implement in a cost-effective way.

The accuracy of the polarization determination will be important for assessing the suppression of the W-pair backgrounds. To illustrate this, consider a potential experiment where the electron beam polarization is $90 \%$ and the positron beam is unpolarized. Suppose an analysis for isolating a new physics signal yields 400 candidate events after analysis cuts, but with no cut on the polarization state. If the right-polarized electron state is chosen, suppose 40 events are observed to survive. This would be an excess of 20 events over what would be expected if the entire sample were due to $\mathrm{W}$-pair backgrounds. The measured left-right asymmetry would be

$$
A_{L R}^{\text {meas }}=0.80 \pm 0.024(\text { stat }) \pm \frac{\delta P}{P} .
$$

The uncertainty on $A_{L R}^{\text {meas }}$ is summarized in Table 1 for 3 possible values of the accuracy of the polarization determination. To achieve a $4 \sigma$ signal will require better than $1 \%$ polarimetry. More precise polarimetry is generally desirable as the beam polarization increases, to assure accurate assessment of the $\mathrm{W}$-pair background.

\section{Beam-beam Effects on Precise Polarimetry}

Beam-beam effects in the collision process can cause significant depolarization due to spin precession and the Sokolov-Ternov spin flip mechanism.12 For a $500 \mathrm{GeV}$ NLC with a luminosity of $6 \cdot 10^{33} \mathrm{~cm}^{-2} \mathrm{~s}^{-1}$, the luminosity-weighted depolarization is estimated to be about $0.15 \%$. For a $1 \mathrm{TeV}$ NLC with a luminosity of $1.4 \cdot 10^{34}$ $\mathrm{cm}^{-2} \mathrm{~s}^{-1}$, it is estimated to be about $1.5 \% .13$ These calculations should be checked 
Table 3. Figures-of-Merit for W-pair Background Suppression.

\begin{tabular}{ccc}
\hline Electron Polarization & Positron Polarization & FOM \\
\hline 0 & 0 & 1.0 \\
0.8 & 0 & 8.7 \\
0.9 & 0 & 17.7 \\
0.8 & 0.5 & 24.5 \\
0.8 & 0.8 & 61.8 \\
0.9 & 0.8 & 103.2 \\
1.0 & 0 & 260. \\
1.0 & 1.0 & 260. \\
\hline
\end{tabular}

Table 4. Dependence of Signal Asymmetry Error on Polarization Error.

\begin{tabular}{ccc}
\hline$\frac{\partial P}{P}$ & $\delta A_{L R}^{\text {meas }}$ & $\begin{array}{c}\text { Significance } \\
\text { of Result }\end{array}$ \\
\hline 0 & $2.4 \%$ & $4.2 \sigma$ \\
$1 \%$ & $2.6 \%$ & $3.8 \sigma$ \\
$2 \%$ & $3.1 \%$ & $3.2 \sigma$ \\
\hline
\end{tabular}

experimentally, however. This can be done with a Compton polarimeter in the extraction line from the IR, by comparing polarization measurements with and without collisions. 1

The extraction line Compton polarimeter measures the total depolarization in the collision process. The luminosity-weighted depolarization is typically onequarter of this 12 This is easily understood for spin precession effects, where the depolarization has a quadratic dependence on the precession angle and one assumes that half the precession occurs before the hard collision. ${ }^{d}$ For example, the depolarization due to the large disruption angles of the beams is

$$
\begin{aligned}
\Delta P_{e} & =\frac{1}{2}\left(\gamma \frac{g-2}{2}\right)^{2}\left[\sigma_{x^{\prime}}^{2}+\sigma_{y^{\prime}}^{2}\right] \\
\Delta P_{e}^{\text {lum }} & \approx \frac{1}{2}\left(\gamma \frac{g-2}{2}\right)^{2}\left[\left(\frac{\sigma_{x^{\prime}}}{2}\right)^{2}+\left(\frac{\sigma_{y^{\prime}}}{2}\right)^{2}\right] \\
\Delta P_{e}^{\text {lum }} & \approx \frac{1}{4} \Delta P_{e},
\end{aligned}
$$

where $\sigma_{x^{\prime}}\left(\sigma_{y^{\prime}}\right)$ is the disrupted $\mathrm{x}(\mathrm{y})$ angular divergence; $\gamma\left(\frac{g-2}{2}\right)$ is the spin precession factor; $\Delta P_{e}$ is the total depolarization and $\Delta P_{e}^{\text {lum }}$ is the luminosity-weighted depolarization.

It is important for IR physicists to include depolarization in their tabulations of beam-beam effects. Extensive tables of beam-beam effects for energy distributions,

${ }_{d}$ This assumption may not be valid if the beams undergo significant betatron oscillations during the collision. In that case, the luminosity-weighted depolarization may be comparable to the total depolarization. 
luminosity distributions and outgoing angular distributions are produced in the design reports for NLC, JLC and TESLA. Surprisingly, depolarization effects are not included. This is presumably due to the lack of depolarization calculations in the beam-beam effect simulation codes used, CAIN 14 and GUINEA-PIG.15 These programs should be improved to include depolarization effects, and depolarization should be included as a key element in tables summarizing beam-beam effects.

At high luminosities, or for the more severe beam disruption experienced in $e^{-} e^{-}$collisions, it becomes difficult to transport the disrupted beam cleanly to the beam dumps. 16 This may lead to an extraction line design that precludes a Compton polarimeter and other beam diagnostics. In this scenario, one will have to rely on a Compton polarimeter before the IP, and possibly utilize SM physics asymmetries for polarimetry as well. By comparing the upstream Compton polarization measurement with that provided by a SM physics asymmetry, the beam-beam depolarization can be determined. This depolarization determination will have greater systematic uncertainty than that achievable with an extraction line Compton polarimeter, for which many sources of systematic error will cancel when determining the amount of depolarization. However, $P_{e}^{\text {lum }}$ and $\Delta P_{e}^{\text {lum }}$ will be more directly determined, perhaps offsetting this disadvantage. Ideally, both extraction line Compton polarimetry and SM physics asymmetry polarimetry will be achievable.

\section{Conclusions}

Precise measurements of SM asymmetries in both $e^{+} e^{-}$and $e^{-} e^{-}$collider modes require better than $1 \%$ polarimetry. Sub-1\% polarimetry may also be required to accurately assess $\mathrm{W}$-pair backgrounds in a discovery search for a new physics signal at an $e^{+} e^{-}$collider.

A Compton polarimeter in the extraction line from the IP is desirable, especially for its ability to accurately measure depolarization effects. SM physics asymmetries are useful for polarimetry when both colliding beams are polarized. For the $e^{+} e^{-}$collider with only the electron beam polarized, the asymmetry in forward $\mathrm{W}$-pairs may also prove useful as a polarimeter. For the $Z^{0}$-factory $e^{+} e^{-}$collider (below W-pair threshold) with no positron polarization, a very precise Compton polarimeter in the extraction line is required.

There is a need to include depolarization in the tables summarizing beam-beam effects. Including depolarization calculations in the simulation programs for beambeam effects will assist this.

\section{Acknowledgements}

I would like to thank Michael Peskin for discussions and comments regarding this paper. I would also like to thank Clemens Heusch for organizing this meeting and for promoting the less conventional aspects of linear colliders. 


\section{References}

1. M. Woods, Int. J. Mod. Phys. A13, 2517 (1998).

2. A. Blondel, Phys. Lett. B202, 145 (1988).

3. F. Cuypers and P. Gambino, Phys. Lett. B388, 211 (1996); F. Cuypers and P. Gambino, PSI-PR-96-27 (1996).

4. Physics and Technology of the Next Linear Collider, SLAC Report 485 (1996); report submitted to Snowmass '96.

5. A.V. Subashiev and J.E. Clendenin, SLAC-PUB-8312 (2000); paper submitted to these proceedings that discusses the possibilities for achieving electron beam polarization of $90 \%$ or greater.

6. D.G. Charlton, e-print hep-ex/9912019 (1999). (See Figure 7 in this paper.)

7. R. Hawkings and K. Monig, DESY 99-157 (1999).

8. M. Swartz, e-print hep-ex/9912026 (1999).

9. A. Czarnecki and W.J. Marciano, e-print hep-ph/0003019 (2000).

10. R. Carr et al., SLAC-PROPOSAL-E-158 (1997).

11. T. Omori, KEK Preprint 98-237 (1999).

12. K. Yokoya and P. Chen, SLAC-PUB-4692, (1988).

13. D. Burke, SLAC NLC Internal Memo, unpublished (1995).

14. P. Chen et al., Nucl. Inst. Meth. A355, 107 (1995).

15. D. Schulte, PhD Thesis U. Hamburg, DESY-TESLA-97-08 (1997).

16. Y.M. Nosochkov and T.O. Raubenheimer, SLAC-PUB-8313 (1999). 